\title{
Dihydro-alpha-lipoic acid binds to human serum albumin at Sudlow I binding site
}

2 Nikola Gligorijević, Vladimir Šukalović, Goran Miljuš, Olgica Nedić, Ana Penezić*

3 Institute for the Application of Nuclear Energy, Department for Metabolism, University of

4 Belgrade, Banatska 31b, 11080 Belgrade

5 Institute of Chemistry, Technology and Metallurgy, University of Belgrade, Njegoševa 12,

611000 Belgrade, Serbia

*Corresponding author

Ana Penezić

11 Institute for the Application of Nuclear Energy, Department for Metabolism

University of Belgrade

Banatska 31b, 11080 Belgrade

E-mail: anap@inep.co.rs

\section{ABSTRACT}

Binding of dihydro-alpha-lipoic acid (DHLA) to human serum albumin (HSA) was characterised in detail in this study. Binding process was monitored by spectroscopic methods and molecular docking approach. HSA binds DHLA with moderate affinity, $0.80 \pm 0.007 \times 10^{4} \mathrm{M}^{-1}$. Spectroscopic data demonstrated that the preferential binding site for DHLA on HSA is IIA (Sudlow I). Hydrogen bonds and electrostatic interactions were identified as the key binding interactions. DHLA binding thermally stabilized HSA, yet it had no effect on HSA structure and its susceptibility to trypsin digestion. Molecular docking confirmed that Sudlow I site accommodated DHLA in a certain conformation in order for binding to occur. Molecular dynamic simulation showed that formed complex is stable. Reported results offer future perspectives for investigations regarding the use of DHLA as a dietary intervention but also raise

27 COVID-19. 


\section{INTRODUCTION}

Human serum albumin (HSA) is the most dominant protein in the circulation, with a referent concentration range from 35 to $50 \mathrm{~g} / \mathrm{L}(522 \mu \mathrm{M}$ to $746 \mu \mathrm{M})$. This is a protein with molecular mass of $67 \mathrm{kDa}$ (Wang, Tian, \& Chang, 2012). Structurally, HSA is composed of three homologous domains (I, II and III), each divided into two subdomains, A and B. Dominant secondary structure motif of HSA is $\alpha$-helix (McLachlan \& Walker, 1977).

HSA has many important functions in the circulation. Due to its high concentration, HSA participates in the osmotic pressure regulation (Lee \& Wu, 2015). Its free Cys34 thiol group (in healthy individuals $70-80 \%$ of Cys 34 thiol group is in a reduced form), makes HSA an important factor for plasma antioxidant capacity, contributing by $80 \%$ to the total plasma thiol amount (Pavićević et al., 2014). HSA is also a general transporter of fatty acids, ions and drugs. Due to its structure, HSA is able to accommodate and bind a variety of small molecules with moderate to high affinities. Two main binding sites for a plethora of different molecules (excluding fatty acids) are located at IIA subdomain or Sudlow I binding site, and IIIA subdomain or Sudlow II binding site. Drugs warfarin and ibuprofen are stereotypical ligands for Sudlow site I and Sudlow site II, respectively (Fasano et al., 2005).

Lipoic acid (LA) is a naturally occurring molecule whose main sources are potato, broccoli and spinach. Humans can also synthetize LA in small amounts. LA is readily absorbed from foods and its oral administration as a drug is a viable therapeutic option, including the treatment of patients with COVID-19 infection (Horowitz \& Freeman, 2020; Zhang \& Liu, 2020). LA supplements are also commercially available, with LA concentrations up to $600 \mathrm{mg}$ per tablet. LA is shown to improve glycemic control, alleviate symptoms of diabetic polyneuropathy and is also effective against toxicity caused by heavy metal poisoning. Antioxidant activity of LA is manifested through ROS scavenging, transition metal ions (e.g., iron and copper) chelating, cytosolic glutathione and vitamin $\mathrm{C}$ levels increase, and oxidative stress damage repair (Zuliani \& Baroni, 2015).

Following cellular uptake, LA is reduced to dihydrolipoic acid (DHLA), which is a very potent reducing agent (Zuliani \& Baroni, 2015). LA has several beneficial effects such as antioxidant, improvement of glycemic control, mitigation of toxicity by heavy metal poisoning and immunomodulatory effects (Salinthone, Yadav, Bourdette, \& Carr, 2008; Smith, Shenvi, Widlansky, Suh, \& Hagen, 2004; Zuliani \& Baroni, 2015).

Although the ability of albumin to bind DHLA is well known (Kawabata \& Packer, 1994), no detailed analysis of this interaction has been reported so far. In the case of bovine albumin (BSA), DHLA was shown to bind at IIIA site (Suji et al., 2008), however no binding experiments in the presence of the specific ligand for this site were performed. Taking into account structural similarity of DHLA and octanoic fatty acid, it was proposed that DHLA binds 
67 to IIA site (Atukeren, Aydin, Uslu, Gumustas, \& Cakatay, 2010), however, IIIA site was also considered (Suji et al., 2008).

Having in mind that DHLA is a very potent antioxidant and its use can alleviate a number of conditions related to oxidative stress, it seemed relevant to elucidate its mode of interaction with HSA, a universal transporter in the circulation. The properties of this interaction, are still unknown and undefined, so the present study aimed to investigate characteristics of the DHLAHSA binding in detail, by using spectroscopic and molecular docking approach.

\section{MATERIALS AND METHODS}

\section{$75 \quad$ Materials}

76 All chemicals used were of analytical grade and were purchased from Sigma (Burlington,

77 Massachusetts, USA). Stock solution of HSA, purchased from Sigma (A-1653) and used without 78 additional purification, was made by dissolving HSA in $10 \mathrm{mM}$ PBS, pH 7.4. The concentration 79 of HSA was determined by using bicinchoninic acid (BCA) assay kit (Thermo Fisher Scientific, Waltham, Massachusetts, USA). Stock solution ( $5 \mathrm{mM}$ ) of DHLA was prepared by suspending DHLA in $10 \mathrm{mM}$ PBS and then adding a small volume of $1 \mathrm{M} \mathrm{NaOH}$ until full clarification of solution was reached (Perricone et al., 1999). Trypsin was purchased from the Institute Torlak (Belgrade, Serbia) as a $0.25 \%$ solution. All experiments were performed in triplicate at room temperature, using $10 \mathrm{mM}$ PBS, $\mathrm{pH} 7.4$, unless otherwise stated.

Binding constant (Ka) of HSA-DHLA complex was determined by recording the quenching of intrinsic fluorescence emission of HSA $(0.4 \mu \mathrm{M})$ in the presence of increasing concentrations of DHLA (from 4 to $35 \mu \mathrm{M}$ ) at $37{ }^{\circ} \mathrm{C}$. Fluorescence spectra were recorded using FluoroMax®-4 spectrofluorometer (Horiba Scientific, Japan). HSA was exciteed at $280 \mathrm{~nm}$ and emission spectra were recorded in the range from 290 to $450 \mathrm{~nm}$. Each spectrum was corrected for the emission of the control that contained only DHLA at particular concentration. The change of the emission intensity at $338 \mathrm{~nm}$ (HSA emission maximum) was used for the calculation of the binding constant. Emission intensity measured for HSA was first corrected for the small inner filter effect of DHLA using the equation:

$$
\mathrm{F}_{\mathrm{c}}=\mathrm{F}_{0} \times 10^{(\mathrm{Aex}+\mathrm{Aem}) / 2}
$$

where $\mathrm{Fc}$ is corrected fluorescence, $\mathrm{F}_{0}$ is measured fluorescence, Aex and Aem are absorbances

97 at excitation and emission wavelengths which are $290 \mathrm{~nm}$ and $338 \mathrm{~nm}$, respectively.

98 Using corrected fluorescence, binding constant between HSA and DHLA was calculated using the following equation: 
100

101

102

103

104

105

106

107

108

109

110

111

112

113

114

115

116

117

118

119

120

121

122

123

124

125

126

127

128

$$
\log \frac{F_{0}-F}{F}=-n \log \frac{1}{[L]-[P] \frac{F_{0}-F}{F_{0}}}+n \log K_{a}
$$

where $\mathrm{F}_{0}$ and $\mathrm{F}$ represent intensities of emission signals of HSA in the absence and in the presence of DHLA, [L] represents the total concentration of ligand (DHLA) and [P] the total concentration of protein (HSA).

Type of quenching, whether it's static (complex formation) or dinamic, was determined by ploting Stern-Volmer (SV) graph and calculating SV quenching constant (Ksv) from it by applying the following equation:

$$
\frac{\mathrm{F}_{0}}{\mathrm{~F}}=1+\mathrm{k}_{\mathrm{q}} \tau_{0}[\mathrm{Q}]=1+\mathrm{K}_{\mathrm{SV}}[\mathrm{Q}]
$$

where $\mathrm{F}_{0}$ and $\mathrm{F}$ are intensities of emission signals without and in the presence of DHLA, $\mathrm{k}_{\mathrm{q}}$ represents the biomolecule quenching rate constant, $\tau 0$ is the average lifetime of the biomolecule without quencher $\left(10^{-8} \mathrm{~s}\right),[\mathrm{Q}]$ is the total concentration of quencher (DHLA). The slope from SV plot represents $\mathrm{K}_{\mathrm{SV}}$. $\mathrm{K}_{\mathrm{SV}}$ was further used for the calculation of $\mathrm{k}_{\mathrm{q}}$.

Thermodynamic parameters of DHLA binding to HSA were calculated by using the same experimental approach as for Ka calculation but at three different temperatures, 25, 30 and 37 ${ }^{\circ} \mathrm{C}$. Calculated binding constants at three temperatures were then used to plot Van't Hoff graph. Enthalpy $(\Delta \mathrm{H})$ and entropy $(\Delta \mathrm{S})$ were calculated from that graph applying the following equation:

$$
\ln \mathrm{Ka}=-\frac{\Delta \mathrm{H}}{\mathrm{RT}}+\frac{\Delta \mathrm{S}}{\mathrm{R}}
$$

where $\mathrm{T}$ is temperature in Kelvins $(\mathrm{K})$ and $\mathrm{R}$ is a universal gas constant $\left(8.314 \mathrm{Jmol}^{-1} \mathrm{~K}^{-1}\right) . \Delta \mathrm{H}$ was calculated from the slope of Van't Hoff graph and $\Delta \mathrm{S}$ from the intercept. The change in Gibbs free energy was calculated from the equation:

$$
\Delta \mathrm{G}=\Delta \mathrm{H}-\mathrm{T} \Delta \mathrm{S}
$$

For specific fluorescence emission changes of 18 Tyr residues or the only Trp214 residue, synchronous fluorescence spectra were recorded on RF-6000 spectrophotometer (Shimadzu, Japan). Spectra were recorded in the range from 280 to $330 \mathrm{~nm}$ with $\Delta \lambda$ of $60 \mathrm{~nm}$ for Trp214 and in the range from 290 to $325 \mathrm{~nm}$ with $\Delta \lambda$ of $15 \mathrm{~nm}$ for Tyr residues. Here, $\Delta \lambda$ represents $\Delta \lambda$ of emission $-\Delta \lambda$ of excitation for each specific residue.

For the confirmation of the specific binding site for DHLA on HSA, site IIA (Sudlow I) on HSA $(0.4 \mu \mathrm{M})$ was blocked using site-specific ligand warfarin $(40 \mu \mathrm{M})$. DHLA $(20$ and $40 \mu \mathrm{M})$ was 
added to this mixture and specific fluorescence emmision of wafarin $(\lambda \mathrm{ex}=310 \mathrm{~nm})$ was recoreded in the range from 340 to $440 \mathrm{~nm}$ (Vasquez, Vu, Schultz, \& Vullev, 2009).

\section{Circular dichroism (CD) spectropolarimetric analysis of HSA-DHLA complex}

The influence of DHLA binding on HSA structure was determined by CD-spectropolarimeter J815 (Jasco, Japan) at room temperature and scan speed of $50 \mathrm{~nm} / \mathrm{min}$. Different concentrations of DHLA were added $(6,15$ and $30 \mu \mathrm{M})$ to HSA $(3 \mu \mathrm{M})$. Both HSA and DHLA stock solutions were dissolved in $10 \mathrm{mM}$ phosphate buffer, $\mathrm{pH}$ 7.4. Tertiary protein structure was analyzed by recording near-UV CD spectra in the range from 260 to $320 \mathrm{~nm}$ using a cell path of $10 \mathrm{~mm}$, while secondary protein structure was monitored by recording a far-UV CD spectra in the range from 185-260 nm using a cell path of $0.5 \mathrm{~mm}$. Spectra obtained for mixtures were corrected for spectra derived from DHLA alone.

\section{UV-VIS analysis of HSA-DHLA complex}

UV-VIS spectra of HSA $(9 \mu \mathrm{M})$ in the presence of DHLA at different concentrations $(9,45$ and $90 \mu \mathrm{M}$ ) were recorded at room temperature using Ultrospec 2000 spectrophotometer (Pharmacia Biotech, Sweden) in the range from 250 to $300 \mathrm{~nm}$. A spectrum of each mixture was corrected for a spectrum obtained for DHLA alone. Also, UV-VIS spectrum of DHLA $(90 \mu \mathrm{M})$ in the presence of HSA $(9 \mu \mathrm{M})$ was recorded in the range from 300 to $450 \mathrm{~nm}$ and corrected for a spectrum obtained for HSA alone.

\section{Temperature stability analysis of HSA-DHLA complex}

Temperature stability of HSA $(0.4 \mu \mathrm{M})$ alone and in the presence of DHLA (40 $\mu \mathrm{M})$ was determined by recording the reduction of fluorescence emission at $338 \mathrm{~nm}$ (emission peak of HSA) and at $335 \mathrm{~nm}$ (emission peak of HSA-DHLA complex), using the same equipment as in the titration experiment. Emission was recorded in the temperature range from 37 to $87^{\circ} \mathrm{C}$ with a temperature increase rate of $2{ }^{\circ} \mathrm{C}$. A mixture was allowed to equilibrate for $1 \mathrm{~min}$ before the measurement at each temperature. The obtained spectra were corrected by subtracting spectra of DHLA alone at each temperature. Results were fitted to sigmoid curves where inflection points represent melting temperatures $(\mathrm{Tm})$.

\section{Proteolytic analysis using trypsin}

For the investigation if DHLA binding affects susceptibility of HSA to trypsin proteolysis, the following experiment was performed at $37^{\circ} \mathrm{C}$. To solutions containing $4 \mu \mathrm{M}$ HSA, alone and in the presence of DHLA $(40 \mu \mathrm{M}), 25 \mu \mathrm{L}$ of $0.25 \%$ trypsin solution was added. The final volume of reaction mixtures was $1 \mathrm{~mL}$. At certain time points $(1,5,10,20$ and $30 \mathrm{~min}) 50 \mu \mathrm{L}$ aliquots were taken from the reaction mixture and PMSF immediately added at the final concentration of $2 \mathrm{mM}$, thus stopping the reaction. Proteolytic fragments of HSA were analyzed by reducing 
SDS-PAGE using a $12 \%$ gel in a standard manner. Gel was stained using Silver Stain Plus Kit (Bio-Rad, Hercules, California, USA).

\section{Docking simulations}

Docking simulations were carried out with Schrodinger Maestro Suite (Schrödinger, LLC, New York, NY, 2018) using crystal structure of HSA complexed with warfarin (PDB code: 2BXD, (Ghuman et al., 2005), obtained from RCSB PDB database (https://www.rcsb.org/). DHLA structure was drawn in ChemDraw program (PerkinElmer Informatics, 2017). All structures were prepared in Maestro software, using default procedures. Up to 20 different docked structures were generated with Induced fit docking protocol (Sherman, Day, Jacobson, Friesner, $\&$ Farid, 2006). The obtained docking structures were examined and the best structure was selected based on the number of receptor-ligand interactions and docking score.

\section{Molecular dynamics (MD) simulations}

MD simulations were done in Schrodinger Desmond software package (Bowers et al., 2006). Selected docked structure for MD was solvated with TIP3P explicit water model, and neutralized via counter ions. Salt solution of $0.15 \mathrm{M} \mathrm{KCl}$ was added. To calculate the interactions between all atoms OPLS 2003 force field was used. For the calculation of the long-range Coulombic interactions, particle-mesh Ewald (PME) method was used, with the cut-off radius of $9 \AA$ for the short-range Van der Waals (VdW) and electrostatic interactions.

During the course of the simulation, constant temperature of $310 \mathrm{~K}$ and a pressure of $1.01235 \mathrm{bar}$ were maintained, using the Nose-Hoover thermostat, and the Martyna Tobias Klein method. 50 ns MD simulation with 2.0 fs step was performed and the collected trajectory used in the MD analysis to asses docking pose and the stability of protein-ligand interactions.

\section{RESULTS AND DISSCUSION}

\section{Binding of DHLA by HSA}

The presence of DHLA quenches intrinsic fluorescence of HSA, as can be seen from Figure 1A. Moreover, a very small blue shift of $3 \mathrm{~nm}$ is observed at the emission maximum of HSA, as the concentration of DHLA increases. These results suggest that HSA binds DHLA and that polarity of the surrounding of Tyr and Trp214 amino acid residues is not significantly altered. Fluorescence quenching can be both dynamic and static (complex formation). In order to determine which type is present here, SV graph was plotted (Figure 1B) and from its slope Ksv was calculated. The obtained SV plot is linear $\left(r^{2}=0.99\right)$, indicating that only one type of quenching occurs in the observed system. Ksv was calculated to be $0.83 \times 10^{4} \mathrm{M}^{-1}$ and the quenching rate constant of the biomolecule, $\mathrm{k}_{\mathrm{q}}$, was calculated to be $0.83 \times 10^{12} \mathrm{M}^{-1}$. Since $\mathrm{k}_{\mathrm{q}}$ is about two orders of magnitude higher than the diffusion rate of biomolecules $\left(10^{10} \mathrm{M}^{-1} \mathrm{~s}^{-1}\right)$, this result strongly suggests the presence of static type of quenching, confirming that HSA binds 
DHLA. From the equation (2) and the plot from Figure $1 \mathrm{C}$, Ka at $37^{\circ} \mathrm{C}$ was calculated to be $0.80 \pm 0.007 \times 10^{4} \mathrm{M}^{-1}$, showing that HSA binds DHLA with moderate affinity.

When Ka was calculated at three different temperatures, its value decreased as the temperature increased. This usually occurs, but is not exclusive, in static type of fluorescence quenching (excluding entropy driven binding) since complex formation is weaker at higher temperatures (Van De Weert \& Stella, 2011). Using the obtained Ka values at three temperatures, thermodynamic parameters were calculated from Van't Hoff plot (Figure 1D). Large negative value of $\Delta \mathrm{H}$ was obtained, $-32 \mathrm{kJmol}^{-1}$ as well as small negative value of $\Delta \mathrm{S}, 29 \mathrm{Jmol}^{-1} \mathrm{~K}^{-1}$. These results indicate that electrostatic interactions, hydrogen bonds and Van der Walls interactions are mainly responsible for complex formation between HSA and DHLA. The change in Gibbs free energy, $\Delta \mathrm{G}$, at $37^{\circ} \mathrm{C}$ was calculated to be $-23 \mathrm{kJmol}^{-1}$.

Synchronous fluorescence spectra can give information about changes in the emission of Tyr and Trp amino acid residues. Since HSA has only one Trp residue, located inside the binding site IIA (Sudlow I) (Salem, Lotfy, Amin, \& Ghattas, 2019), information from synchronous spectra provides insight into the binding place for certain ligand. In the presence of increasing concentrations of DHLA, Trp specific emission spectrum was significantly quenched (Figure 2A), while that originating from Tyr was reduced to a very small extent (Figure 2B). Considering the position of the Trp residue in HSA, this result strongly indicated that the binding site for DHLA is located in IIA subdomain (Sudlow I). To confirm this, DHLA was added to HSA in the presence of warfarin, and the change in warfarin fluorescence was recorded. When bound to HSA, warfarin fluorescence intensity increases at its emission maximum (Figure 2C). This is a usual consequence of ligand binding to a protein, since the ligand becomes shielded from water and located in a more hydrophobic environment (Liang, Tajmir-Riahi, \& Subirade, 2008). Similar observation was recorded in the case of phycocyanobilin (PCB) binding to HSA that occurs at both IIA and IB subdomains of HSA (Minic et al., 2015). Binding of warfarin to HSA is well characterized with the affinity constant of about $10^{5} \mathrm{M}^{-1}$ (Li et al., 2014). As warfarin specifically binds to Sudlow I site on HSA, it is used to block this site in the studies aimed to locate the exact binding site for other ligands (Petitpas, Bhattacharya, Twine, East, \& Curry, 2001). Results shown in Figure 2C indicate that the emission spectrum of warfarin remains the same in the presence of DHLA (at two concentrations), confirming that binding site IIA on HSA remains occupied by warfarin, thus suggesting that this site is the preferential binding site for DHLA. Even in equimolar concentrations of DHLA and warfarin, fluorescence spectrum of warfarin remains unaltered, indicating that HSA binding affinity for DHLA is lower than for warfarin, which is in agreement with the calculated Ka for HSA-DHLA complex. Having this in mind, it is noteworthy to mention a current pandemic situation with COVID-19 and its potential treatment with alpha lipoic acid (Horowitz \& Freeman, 2020; Zhang \& Liu, 2020). It was proposed that LA blocks NF- $\kappa \mathrm{B}$ and cytokine formation, and thus alleviates cytokine storm syndrome in critically ill patients (Horowitz \& Freeman, 2020). Since warfarin and its 
derivatives are commonly used as anticoagulants (also included in the therapy of severe cases with COVID-19) which preferentially bind to Sudlow I site on HSA, it is questionable whether LA treatment of patients infected with virus Sars-CoV-2 is sufficiently beneficial if they also receive warfarin.

Proteins absorb light in the UV region at about $280 \mathrm{~nm}$ due to the presence of aromatic amino acid residues and changes in the absorption spectrum of a protein in this region may occur as a consequence of changes in the polarity of the environment close to these residues. Figure $3 \mathrm{~A}$ shows that the UV-VIS absorption spectrum of HSA does not change in the presence of increasing concentrations of DHLA, indicating that no significant conformational change of HSA occurs, thus confirming the results obtained by spectrofluorimetry (Figure 3A). On the other hand, the absorption spectrum of DHLA shows both blue shift of its peak and the reduction of its intensity in the presence of HSA (Figure 3B). Similar effect was previously observed upon DHLA binding to fibrinogen (Gligorijević, Šukalović, Penezić, \& Nedić, 2020) and upon binding of 2-amino-6-hydroxy-4-(4-N,N-dimethylaminophenyl)-pyrimidine-5-carbonitrile to BSA (Suryawanshi, Walekar, Gore, Anbhule, \& Kolekar, 2016). Change in the absorption spectrum of DHLA is an additional proof that it forms a complex with HSA.

\section{Protein structure is often affected by ligand binding. Some ligands induce more ordered} structure, others more disordered, and some have no effect. HSA contains only $\alpha$-helixes as elements of the secondary structure. It was shown that binding of PCB and amoxicillin to HSA increase its content of $\alpha$-helixes (Radibratovic et al., 2016; Yasmeen, Riyazuddeen, \& Rabbani, 2017), while binding of plumbagin, safranal and crocin decrease it (Qais, Husain, Khan, Ahmad, \& Hassan, 2020; Salem et al., 2019). The obtained far-UV CD spectra of HSA (Figure 4A) show a typical signal of the protein where $\alpha$-helixes are dominant, with characteristic negative wide peak in the range from 209 to $220 \mathrm{~nm}$. As it can be seen from this figure, no significant change in the secondary structure of HSA occurs upon binding of DHLA, even when the concentration of DHLA is ten times larger than HSA. Tertiary structure of HSA is also unaltered due to DHLA binding since near-UV CD spectra are practically the same in pre presence of all tested DHLA concentrations (Figure 4B).

\section{Stability of HSA-DHLA complex}

Factors that may affect melting point of a protein, besides its structure, include the presence of bound molecules as well as their structure. When a complex forms, new interactions establish that may contribute to altered thermal stability of a protein. In the case of HSA, free protein has Tm of approximately $62{ }^{\circ} \mathrm{C}$, while bound fatty acids increase its thermal stability reaching Tm from 64 to $72{ }^{\circ} \mathrm{C}$ (Lang \& Cole, 2015). Certain ligands, such as PCB and embelin, also increase thermal stability of HSA (Radibratovic et al., 2016; Yeggoni, Rachamallu, \& Subramanyam, 
2016). On the contrary, some drugs, such as amoxicillin, decrease thermal stability of HSA upon binding (Yasmeen et al., 2017). Commercial HSA used in this study had Tm of $68{ }^{\circ} \mathrm{C}$. In the presence of DHLA, Tm of HSA increases to $70{ }^{\circ} \mathrm{C}$ (Figure 4C). Even though DHLA didn't change the structure of HSA significantly upon binding (Figures 4A and 4B), it seems that new interactions in this complex additionally thermally stabilized the protein.

Increased Tm of HSA in the presence of DHLA indicates that rigidness in the protein structure increases, which may affect its susceptibility to proteolytic cleavage. In order to be proteolyzed, peptide bonds in the protein need to be flexible and exposed enough to enable its accurate accommodation in the active site of a protease. Some ligands, such as bilirubin, reduce the susceptibility of HSA to cleavage by trypsin (Sjödin, Hansson, \& Sjöholm, 1977). According to the results of this study, it seems that DHLA, although it thermally stabilizes HSA, has no significant effect on HSA proteolysis by trypsin (Figure 4D). Thus, it may be expected that the formation of HSA-DHLA complex will not have significant (if any) effect on the protein halflife in circulation in respect to proteolysis. The first and the dominant fragment of HSA resulting from proteolysis by trypsin is the one at about $50 \mathrm{kDa}$, while other fragments with lower concentrations and molecular masses appear later. This finding is in accordance with the already published data (Radibratovic et al., 2016).

\section{Molecular modeling}

Binding site IIA consists of a binding pocket deeply embedded in the core of the subdomain that is formed by all six helices of the subdomain and the loop-helix residues 148-154 of IB (Ghuman et al., 2005). Pocket interior is predominantly hydrophobic, apart from the two clusters of polar residues (Tyr150, His242, Arg257 and Lys195; Lys199, Arg218 and Arg222).

Induced fit docking simulation results have shown that DHLA binds to HSA BS II site (Figure 5). The energetically most favorable conformation of the docked pose has showed that the key interactions are salt bridges formed by DHLA carboxyl group with Arg18 and Arg222 of HSA, followed by hydrogen bonds formed between DHLA sulfhydryl group and Arg257, Ser287 (Figure 5). Molecular docking analysis suggested that DHLA binds at Sudlow I site in a defined conformation, thus favoring interactions with specific amino residues. Having in mind that DHLA has high torsional flexibility due to nine dihedral angles which give many possible rotamers (Vigorito, Calabrese, Paltanin, Melandri, \& Maris, 2017), a recorded change in the absorption spectrum (Figure $3 \mathrm{~B}$ ) could point to a DHLA-conformational shift towards rotamers with the highest probability of being bound to HSA.

To verify docking simulation results, DHLA-HSA interactions were monitored throughout $50 \mathrm{~ns}$ molecular dynamic simulation. MD starting point was the best conformation obtained in docking stage. The obtained MD trajectory was analyzed both in terms of complex stability and the persistence of key DHLA-HSA interactions over simulation time period. 
The observed RMSD values for HSA show that simulation has equilibrated, fluctuations fall within $1-2.5 \AA$ range suggesting minor conformational changes during simulation (Figure 6A).

310

311

312

313

314

315

316

317

318

319

320

321

322

323

324

325

326

327

328

329

330

331

332

333

334

335

336

337

338

339

340

341

342

343

344

Monitored HSA-DHLA interactions showed that key interaction is a salt bridge formed between DHLA carboxyl group and Arg218. This interaction is present over 93\% of simulation time, making it crucial for DHLA binding to HSA and it also indicates correct orientation of DHLA inside the BS. Salt bridge with Arg218 is reinforced by interaction with Arg222 (42\% of simulation time). Once DHLA is in correct position in the BS, additional hydrogen bonds between sulfhydryl group and Ser287, Arg257 are established. Those hydrogen bonds are maintained for a 10\% (Ser287) and 7\% (Arg257) of total simulation time (Figure 6B). All other interactions are observed for less than $5 \%$ of total simulation time (Supplementary Figure 1).

HSA can be modified by oxidation, and slight structural alterations, resulting from this chemical modification, cause an impairment of HSA functions, including its ligand binding ability (Kawakami et al., 2006). Redox state of Cys34 on HSA can also influence its binding properties (Oettl \& Stauber, 2007). Considering its high concentration and the capacity to bind wide range of drugs, changes in binding properties of HSA may have a significant impact on pharmacokinetic and pharmacodynamic (PKPD) characteristics of prescribed drugs. DHLA was shown to be able to protect serum albumin from glycation (Kawabata \& Packer, 1994), methylglyoxal modification (Sadowska-Bartosz, Galiniak, \& Bartosz, 2014) and it can protect Cys34 from oxidation (Atukeren et al., 2010). Thus, by binding to HSA, DHLA can directly protect HSA from oxidation and, at the same time, keep the binding and antioxidative properties of HSA unaltered. Since it is used as a food supplement, a detailed PKPD knowledge on DHLA is very important, including information on its binding partners in the circulation. Besides HSA, fibrinogen was also shown to bind DHLA with a similar affinity (Gligorijević et al., 2020).

\section{CONCLUSION}

The obtained results describe in detail the binding of DHLA to HSA for the first time.

Experimental results have shown that binding site IIA or Sudlow I is the preferential binding site for DHLA. Molecular docking analysis and dynamics confirmed the ability of Sudlow I site to accommodate DHLA and that the formed complex is stable. The binding of DHLA doesn't alter significantly the structure of HSA, although it stabilizes the protein itself to some extent. HSA susceptibility to proteolytic cleavage by trypsin remains the same in the presence of DHLA, thus no change of HSA half-life in the circulation (regarding proteolysis) is expected. The reported results expand the knowledge on PKPD properties of DHLA and offer a future perspective for further investigations regarding the use of DHLA as a dietary intervention. Furthermore, the obtained results raise concerns whether alpha-lipoic acid and DHLA are sufficiently beneficial as a part of the proposed treatment protocol of patients with COVID-19 who are receiving warfarin therapy as well, due to their competitive binding and lower affinity of HSA for these antioxidants than for warfarin. 
Acknowledgments: This research was funded the Ministry of Education, Science and Technological Development of the Republic of Serbia, contract numbers: 451-03-68/202014/200019 and 451-03-68/2020-14/200026. There is no conflict of interest regarding this study.

\section{REFERENCES}

Atukeren, P., Aydin, S., Uslu, E., Gumustas, M. K., \& Cakatay, U. (2010). Redox homeostasis of albumin in relation to alpha-lipoic acid and dihydrolipoic acid. Oxidative Medicine and Cellular Longevity, 3(3), 206-213. https://doi.org/10.4161/oxim.3.3.11786

Bowers, K. J., Chow, E., Xu, H., Dror, R. O., Eastwood, M. P., Gregerson, B. A., ... Shaw, D. E. (2006). Scalable Algorithms for Molecular Dynamics Simulations on Commodity Clusters. In SC '06: Proceedings of the 2006 ACM/IEEE Conference on Supercomputing (pp. 4343). Tampa. https://doi.org/10.1109/SC.2006.54

Fasano, M., Curry, S., Terreno, E., Galliano, M., Fanali, G., Narciso, P., ... Ascenzi, P. (2005). The extraordinary ligand binding properties of human serum albumin. IUBMB Life, 57(12), 787-796. https://doi.org/10.1080/15216540500404093

Ghuman, J., Zunszain, P. A., Petitpas, I., Bhattacharya, A. A., Otagiri, M., \& Curry, S. (2005). Structural basis of the drug-binding specificity of human serum albumin. Journal of Molecular Biology, 353(1), 38-52. https://doi.org/10.1016/j.jmb.2005.07.075

Gligorijević, N., Šukalović, V., Penezić, A., \& Nedić, O. (2020). Characterisation of the binding of dihydro-alpha-lipoic acid to fibrinogen and the effects on fibrinogen oxidation and fibrin formation. International Journal of Biological Macromolecules, 147, 319-325. https://doi.org/10.1016/j.ijbiomac.2020.01.098

Horowitz, R. I., \& Freeman, P. R. (2020). Three novel prevention , diagnostic , and treatment options for COVID-19 urgently necessitating controlled randomized trials. Medical Hypotheses, 143, 109851. https://doi.org/10.1016/j.mehy.2020.109851

Kawabata, T., \& Packer, L. (1994). $\alpha$-Lipoate can protect against glycation of serum albumin, but not low density lipoprotein. Biochemical and Biophysical Research Communications. https://doi.org/10.1006/bbrc.1994.2154

Kawakami, A., Kubota, K., Yamada, N., Tagami, U., Takehana, K., Sonaka, I., ... Hirayama, K. (2006). Identification and characterization of oxidized human serum albumin: A slight structural change impairs its ligand-binding and antioxidant functions. FEBS Journal, 273(14), 3346-3357. https://doi.org/10.1111/j.1742-4658.2006.05341.x

Lang, B. E., \& Cole, K. D. (2015). Unfolding properties of recombinant human serum albumin products are due to bioprocessing steps. Biotechnology Progress, 31(1), 62-69. https://doi.org/10.1002/btpr.1996

Lee, P., \& Wu, X. (2015). Review: Modifications of Human Serum Albumin and Their Binding Effect. Current Pharmaceutical Design, 21(14), 1862-1865. https://doi.org/10.2174/1381612821666150302115025 
Li, Q., Yang, W., Qu, L., Qi, H.-Y., Huang, Y., \& Zhang, Z. (2014). Interaction of Warfarin with Human Serum Albumin and Effect of Ferulic Acid on the Binding. Journal of Spectroscopy, 2014, Artical ID 834501. https://doi.org/10.1155/2014/834501

Liang, L., Tajmir-Riahi, H. A., \& Subirade, M. (2008). Interaction of $\beta$-Lactoglobulin with resveratrol and its biological implications. Biomacromolecules, 9(1), 50-56. https://doi.org/10.1021/bm700728k

McLachlan, A. D., \& Walker, J. E. (1977). Evolution of serum albumin. Journal of Molecular Biology, 112(4), 543-558. https://doi.org/10.1016/S0022-2836(77)80163-0

Minic, S. L., Milcic, M., Stanic-Vucinic, D., Radibratovic, M., Sotiroudis, T. G., Nikolic, M. R., \& Velickovic, T. Ć. (2015). Phycocyanobilin, a bioactive tetrapyrrolic compound of bluegreen alga Spirulina, binds with high affinity and competes with bilirubin for binding on human serum albumin. RSC Advances, 5(76), 61787-61798. https://doi.org/10.1039/c5ra05534b

Oettl, K., \& Stauber, R. E. (2007). Physiological and pathological changes in the redox state of human serum albumin critically influence its binding properties. British Journal of Pharmacology, 151(5), 580-590. https://doi.org/10.1038/sj.bjp.0707251

Pavićević, I. D., Jovanović, V. B., Takić, M. M., Penezić, A. Z., Aćimović, J. M., \& Mandić, L. M. (2014). Fatty acids binding to human serum albumin: Changes of reactivity and glycation level of Cysteine-34 free thiol group with methylglyoxal. Chemico-Biological Interactions, 224, 42-50. https://doi.org/10.1016/j.cbi.2014.10.008

Perricone, N., Nagy, K., Horváth, F., Dajkó, G., Uray, I., \& Zs.-Nagy, I. (1999). Alpha lipoic acid (ALA) protects proteins against the hydroxyl free radical-induced alterations: Rationale for its geriatric topical application. Archives of Gerontology and Geriatrics, 29(1), 45-56. https://doi.org/10.1016/S0167-4943(99)00022-9

Petitpas, I., Bhattacharya, A. A., Twine, S., East, M., \& Curry, S. (2001). Crystal structure analysis of warfarin binding to human serum albumin. Anatomy of drug site I. Journal of Biological Chemistry, 276(25), 22804-22809. https://doi.org/10.1074/jbc.M100575200

Qais, F. A., Husain, F. M., Khan, R. A., Ahmad, I., \& Hassan, I. (2020). Deciphering the interaction of plumbagin with human serum albumin: A combined biophysical and molecular docking study. Journal of King Saud University - Science, 32(6), 2854-2862. https://doi.org/10.1016/j.jksus.2020.07.008

Radibratovic, M., Minic, S., Stanic-Vucinic, D., Nikolic, M., Milcic, M., \& Velickovic, T. C. (2016). Stabilization of human serum albumin by the binding of phycocyanobilin, a bioactive chromophore of blue-green alga spirulina: Molecular dynamics and experimental study. PLoS ONE, 11(12), e0167973. https://doi.org/10.1371/journal.pone.0167973

Sadowska-Bartosz, I., Galiniak, S., \& Bartosz, G. (2014). Kinetics of glycoxidation of bovine serum albumin by methylglyoxal and glyoxal and its prevention by various compounds. Molecules, 19(4), 4880-4896. https://doi.org/10.3390/molecules 19044880 
Salem, A. A., Lotfy, M., Amin, A., \& Ghattas, M. A. (2019). Characterization of human serum albumin's interactions with safranal and crocin using multi-spectroscopic and molecular docking techniques. Biochemistry and Biophysics Reports, 20(September), 100670. https://doi.org/10.1016/j.bbrep.2019.100670

Salinthone, S., Yadav, V., Bourdette, D., \& Carr, D. (2008). Lipoic Acid: A Novel Therapeutic Approach for Multiple Sclerosis and Other Chronic Inflammatory Diseases of the CNS. Endocrine, Metabolic \& Immune Disorders-Drug Targets, 8(2), 132-142. https://doi.org/10.2174/187153008784534303

Sherman, W., Day, T., Jacobson, M. P., Friesner, R. A., \& Farid, R. (2006). Novel procedure for modeling ligand/receptor induced fit effects. Journal of Medicinal Chemistry, 49(2), 534553. https://doi.org/10.1021/jm050540c

Sjödin, T., Hansson, R., \& Sjöholm, I. (1977). Isolation and identification of a trypsin-resistant frag- ment of human serum albumin with b1lirubin- and drug- binding properties*. Biochimica et Biophysica Acta, 494, 61-75. https://doi.org/10.1016/0005-2795(77)90135-0

Smith, A. R., Shenvi, S. V., Widlansky, M., Suh, J. H., \& Hagen, T. M. (2004). Lipoic Acid as a Potential Therapy for Chronic Diseases Associated with Oxidative Stress. Current Medicinal Chemistry, 11(9), 1135-1146. https://doi.org/10.2174/0929867043365387

Suji, G., Khedkar, S. A., Singh, S. K., Kishore, N., Coutinho, E. C., Bhor, V. M., \& Sivakami, S. (2008). Binding of lipoic acid induces conformational change and appearance of a new binding site in methylglyoxal modified serum albumin. Protein Journal, 27(4), 205-214. https://doi.org/10.1007/s10930-008-9126-3

Suryawanshi, V. D., Walekar, L. S., Gore, A. H., Anbhule, P. V., \& Kolekar, G. B. (2016). Spectroscopic analysis on the binding interaction of biologically active pyrimidine derivative with bovine serum albumin. Journal of Pharmaceutical Analysis, 6(1), 56-63. https://doi.org/10.1016/j.jpha.2015.07.001

Van De Weert, M., \& Stella, L. (2011). Fluorescence quenching and ligand binding: A critical discussion of a popular methodology. Journal of Molecular Structure, 998(1-3), 144-150. https://doi.org/10.1016/j.molstruc.2011.05.023

Vasquez, J. M., Vu, A., Schultz, J. S., \& Vullev, V. I. (2009). Fluorescence enhancement of warfarin induced by interaction with $\beta$-cyclodextrin. Biotechnology Progress, 25(4), 906914. https://doi.org/10.1002/btpr.188

Vigorito, A., Calabrese, C., Paltanin, E., Melandri, S., \& Maris, A. (2017). Regarding the torsional flexibility of the dihydrolipoic acid's pharmacophore: 1,3-Propanedithiol. Physical Chemistry Chemical Physics, 19(1), 496-502. https://doi.org/10.1039/c6cp05606g

Wang, R. E., Tian, L., \& Chang, Y.-H. (2012). A Homogeneous Fluorescent Sensor for Human Serum Albumin. Journal of Pharmaceutical and Biomedical Analysis, 63, 165-169. https://doi.org/10.1016/j.jpba.2011.12.035. 
Yasmeen, S., Riyazuddeen, \& Rabbani, G. (2017). Calorimetric and spectroscopic binding studies of amoxicillin with human serum albumin. Journal of Thermal Analysis and Calorimetry, 127(2), 1445-1455. https://doi.org/10.1007/s10973-016-5555-y

Yeggoni, D. P., Rachamallu, A., \& Subramanyam, R. (2016). Protein stability, conformational change and binding mechanism of human serum albumin upon binding of embelin and its role in disease control. Journal of Photochemistry and Photobiology B: Biology, 160, 248259. https://doi.org/10.1016/j.jphotobiol.2016.04.012

Zhang, L., \& Liu, Y. (2020). Potential interventions for novel coronavirus in China: A systematic review. Journal of Medical Virology, 92(5), 479-490. https://doi.org/10.1002/jmv.25707

Zuliani, C., \& Baroni, L. (2015). Antioxidants for the Prevention and Treatment of Multiple Sclerosis: An Overview. In R. R. Watson \& V. R. Preedy (Eds.), Bioactive Nutraceuticals and Dietary Supplements in Neurological and Brain Disease: Prevention and Therapy (First, pp. 341-353). Elsevier Inc. https://doi.org/10.1016/B978-0-12-411462-3.00035-7

\section{FIGURE LEGENDS}

Figure 1. Binding analysis of HSA and DHLA using spectrofluorimetry. Fluorescence emission spectra of HSA (excited at $280 \mathrm{~nm}$ ) in the presence of increasing concentrations of DHLA (A). Stern-Volmer plot (B) and plot used for the determination of the binding constant between HSA and DHLA (C) obtained using fluorescence emission maximum of HSA at $338 \mathrm{~nm}$. Van't Hoffs graph obtained by calculating the binding constant between HSA and DHLA at three different temperatures (D).

Figure 2. Determination of a binding site of DHLA on HSA. Synchronous fluorescence spectra of HSA with $\Delta \lambda=60 \mathrm{~nm}$ for $\operatorname{Trp}(\mathrm{A})$ and $\Delta \lambda=15 \mathrm{~nm}$ for Tyr (B) in the presence of increasing concentrations of DHLA. Fluorescence emission spectra of warfarin (excited at $280 \mathrm{~nm}$ ) in the absence and in the presence of HSA, as well as in the presence of HSA and DHLA at warfarin/DHLA molar ratios of 2/1 and 1/1 (C).

Figure 3. Analysis of structural alterations of HSA and DHLA due to mutual binding. Far-UV $\mathrm{CD}(\mathrm{A})$ and near-UV CD (B) spectra of HSA alone and in the presence of increasing concentrations of DHLA. UV absorption spectra of HSA alone and in the presence of increasing concentrations of DHLA (C). UV-VIS absorption spectra of DHLA alone and in the presence of HSA (D).

Figure 4. Analysis of temperature stability of HSA alone and in the presence of DHLA (A). Analysis of HSA digestion by trypsin in the absence (lanes 1-5, samples taken after 1, 5, 10, 20 and 30 min of proteolysis) and in the presence of DHLA (lanes 6-10) by reducing SDS-PAGE on $12 \%$ gel (B). 
492 Figure 5. An overview of HSA with DHLA docked into BS II . Domains are color coded and 493 represented as secondary structure ribbons. BS II composition and key interactions diagram . All 494 amino acid residues in close contact with DHLA are displayed, with key amino acid residues 495 marked.

496 Figure 6. HSA and DHLA RMSD plot (A) and the observed key interactions during $50 \mathrm{~ns}$ 497 simulation time (B).

498 Supplementary Figure 1. Summary of DHLA-HSA interactions observed during 50 ns 499 simulation time. Each orange line represents one established interaction during $1 \mathrm{~ns}$ time frame. 
A

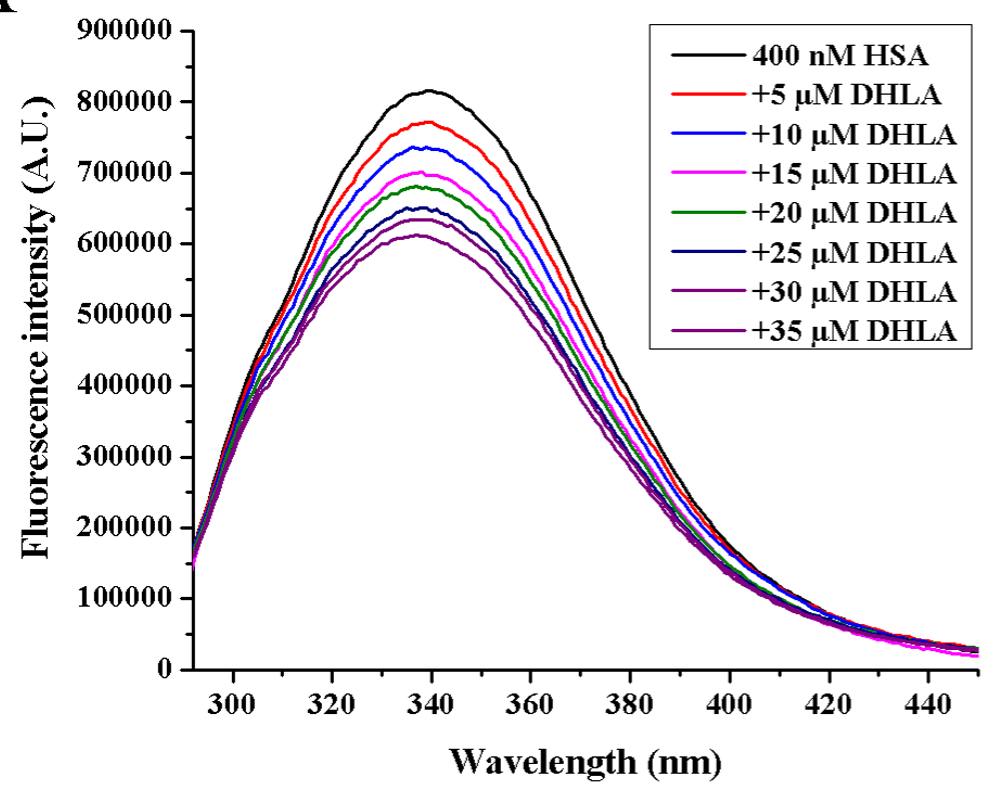

C

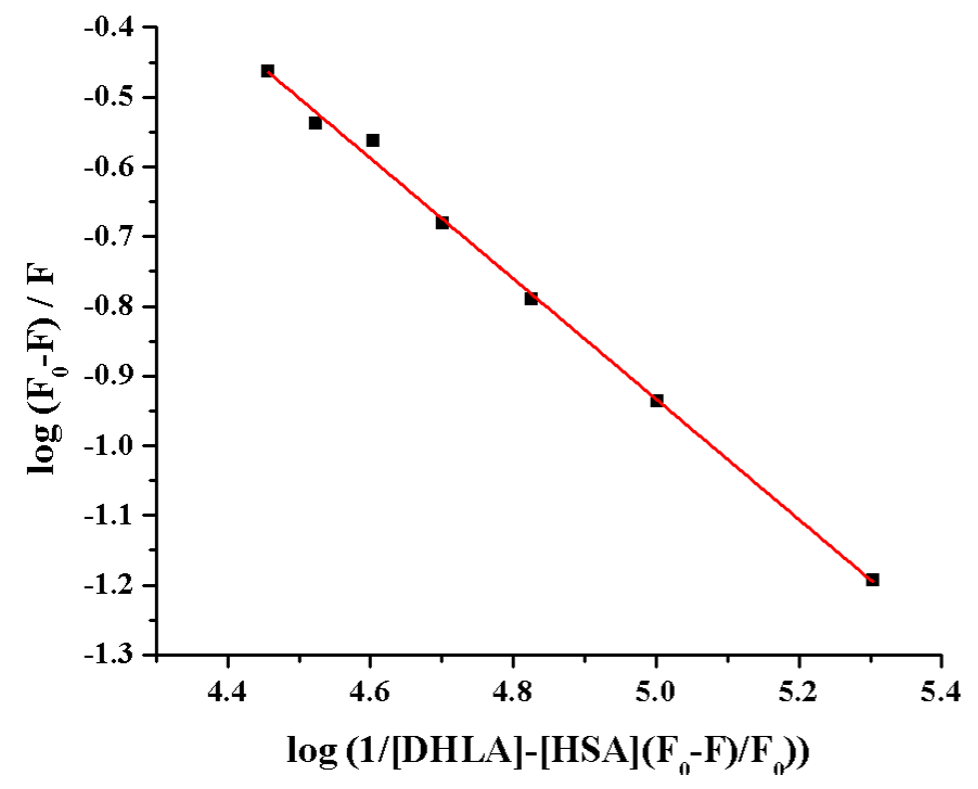

B

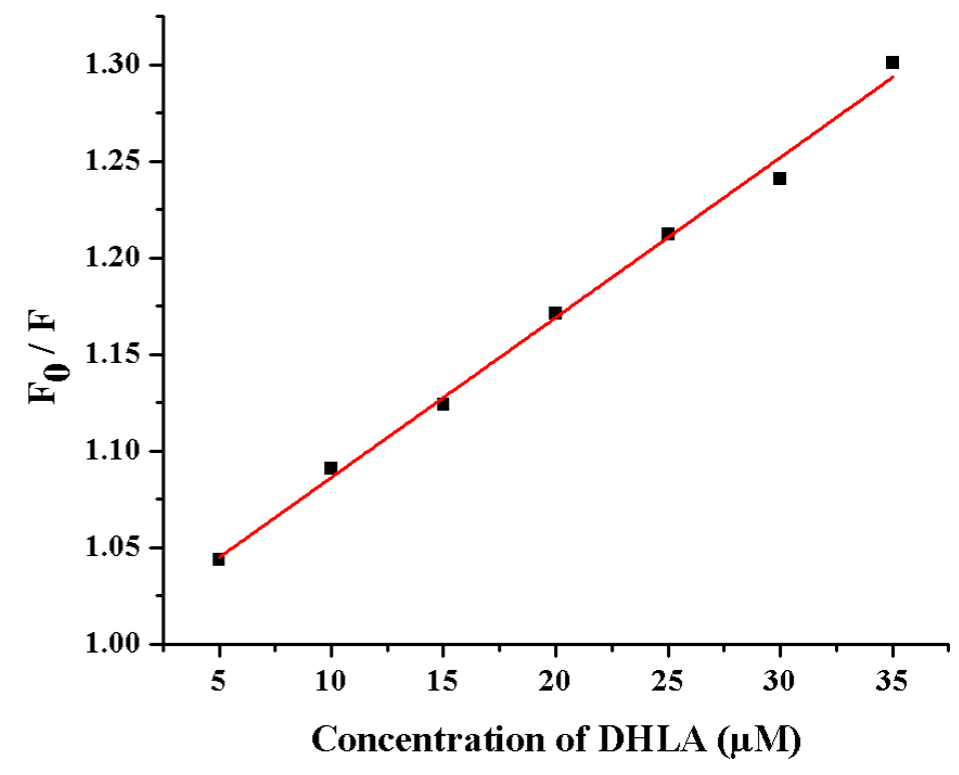

D

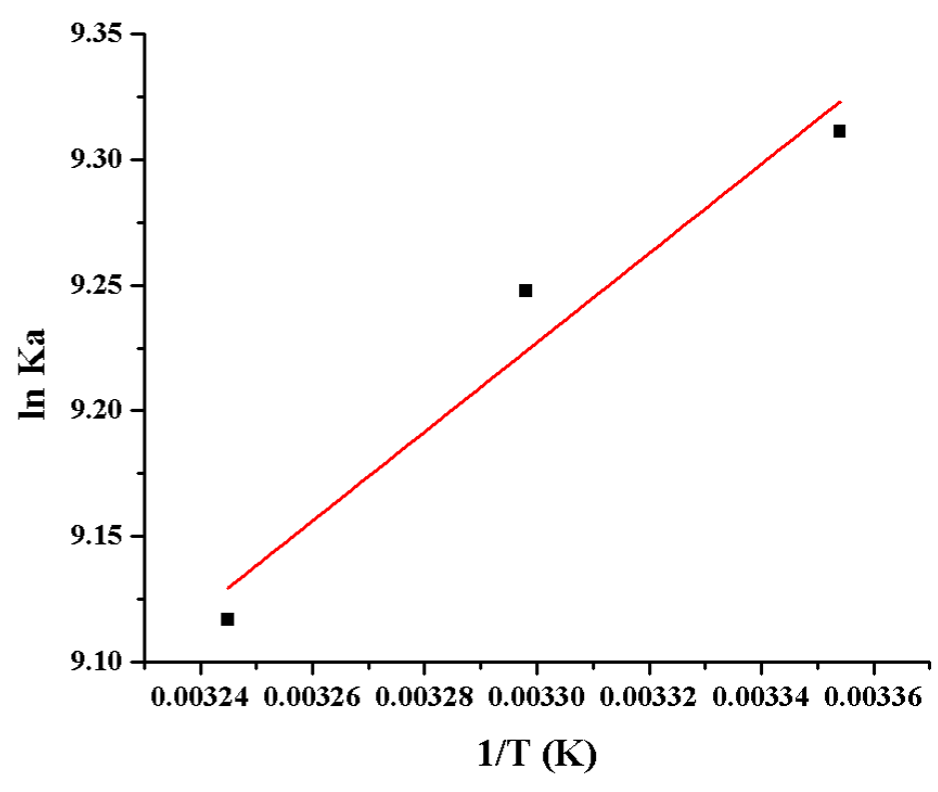


A

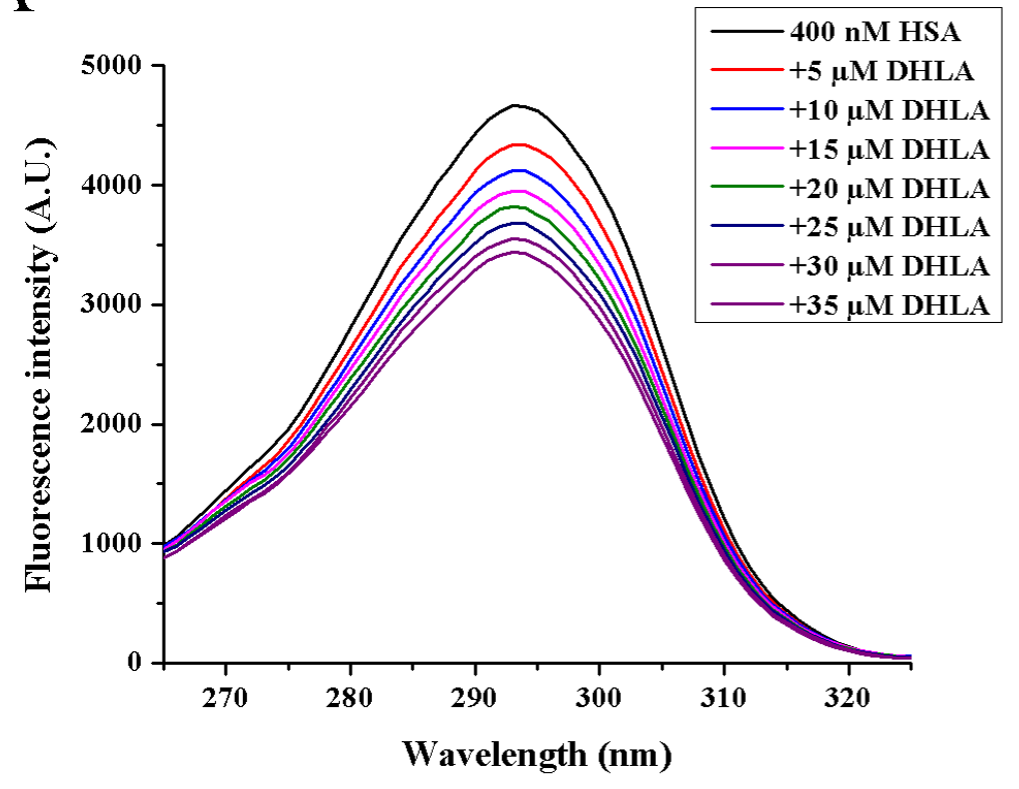

B

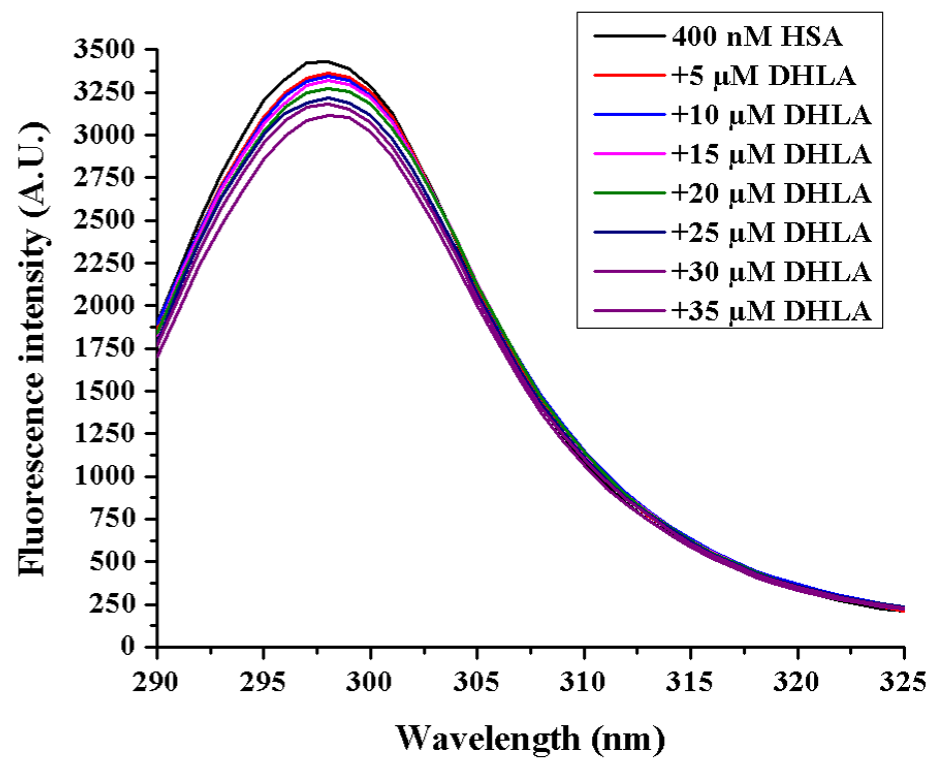

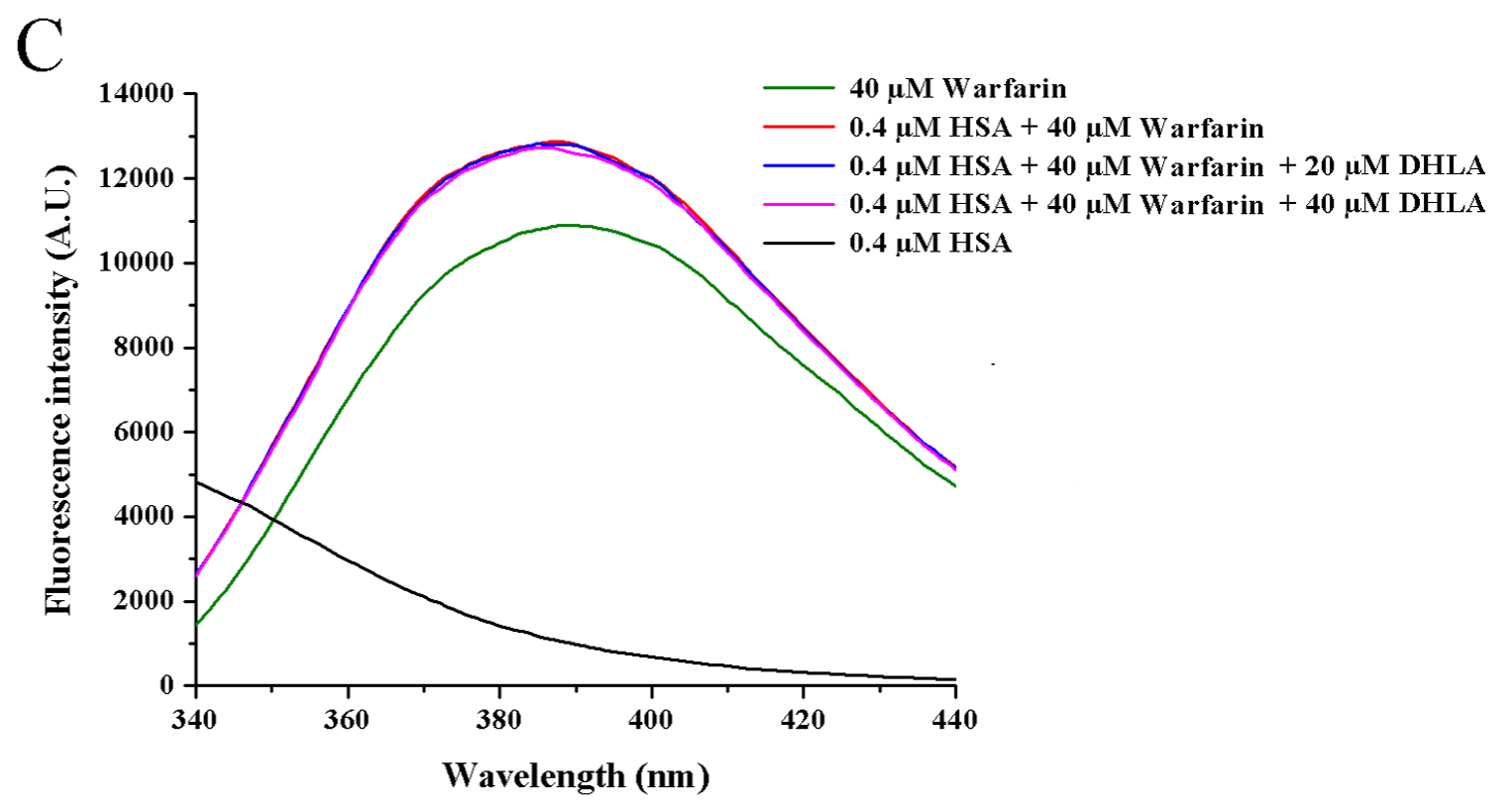


A

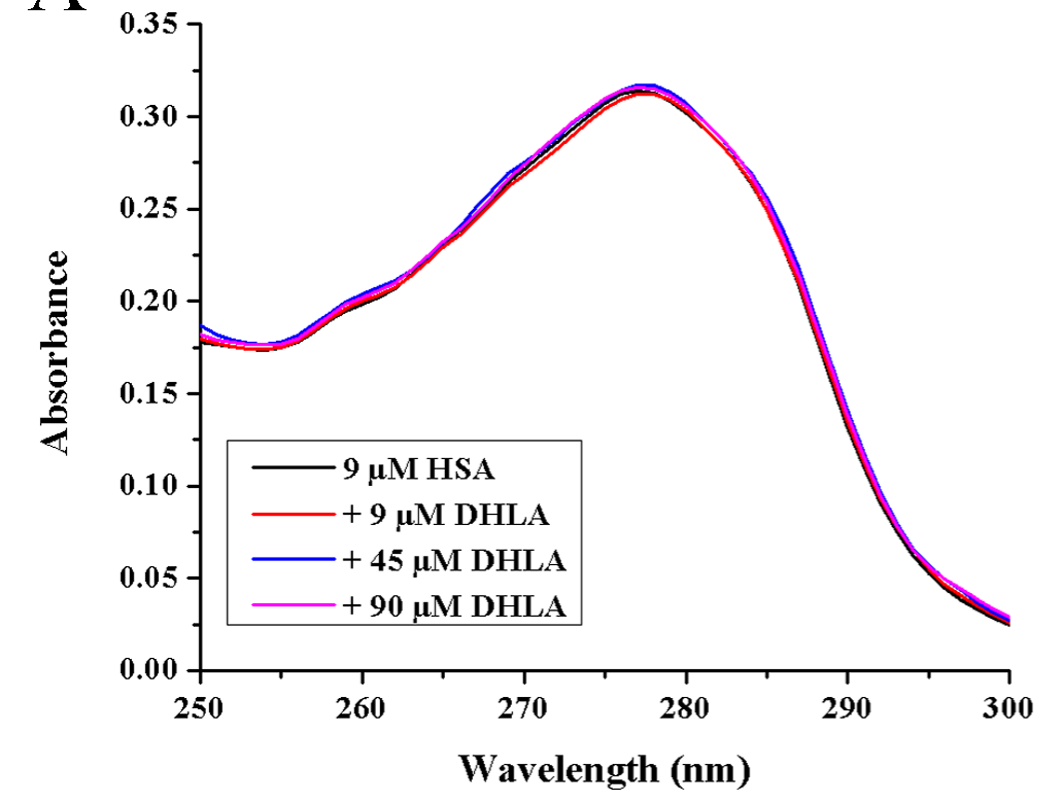

B

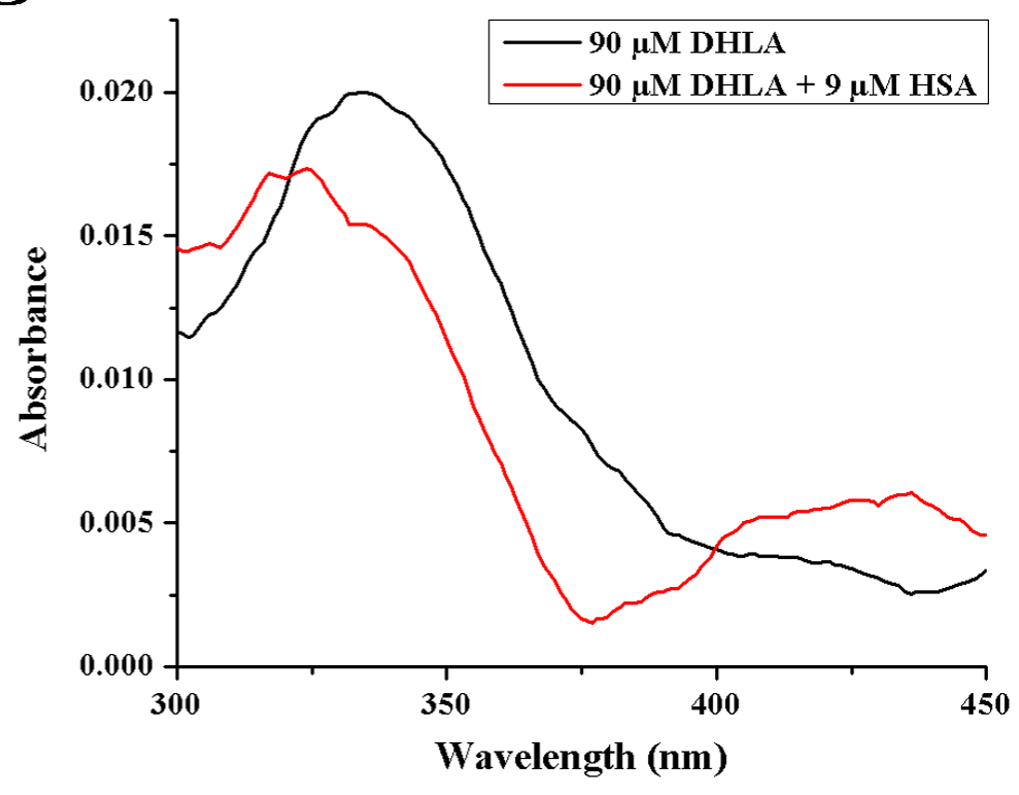


A

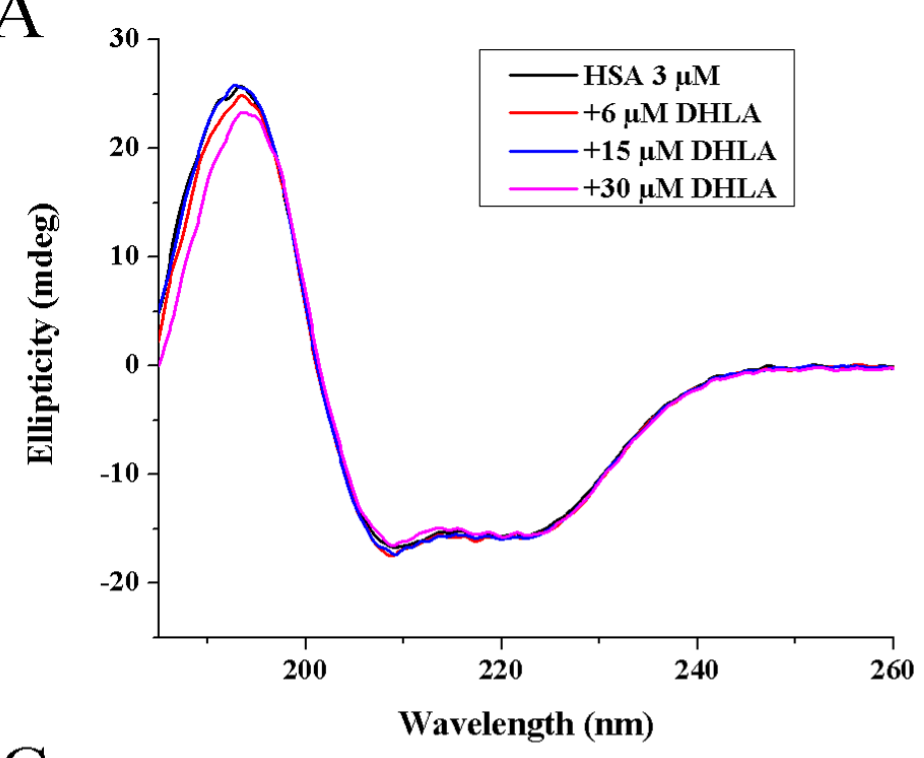

C
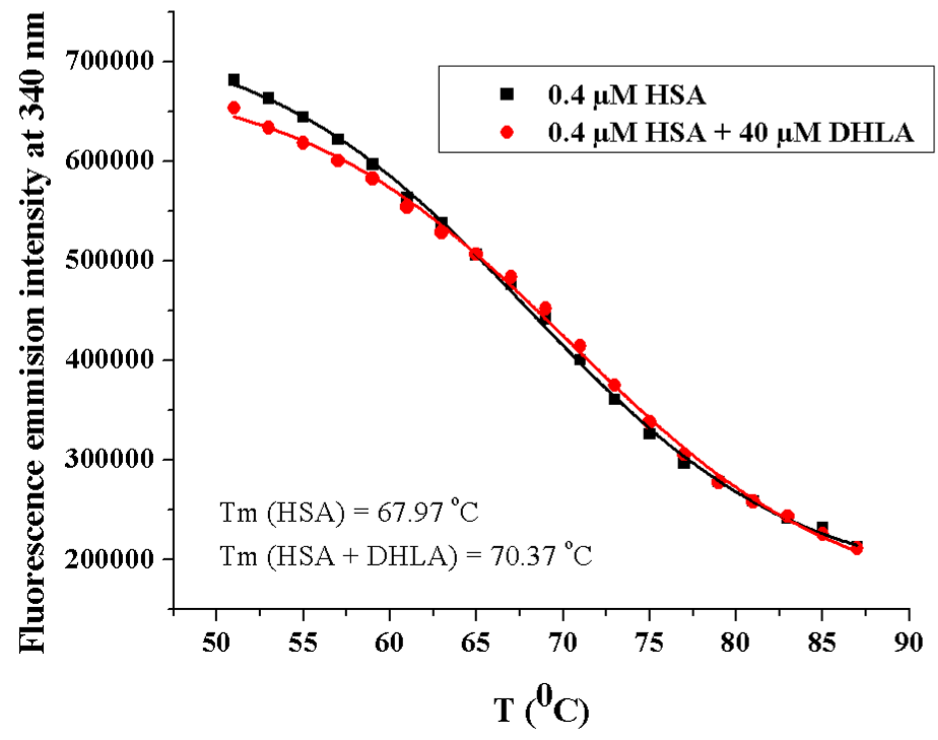

B

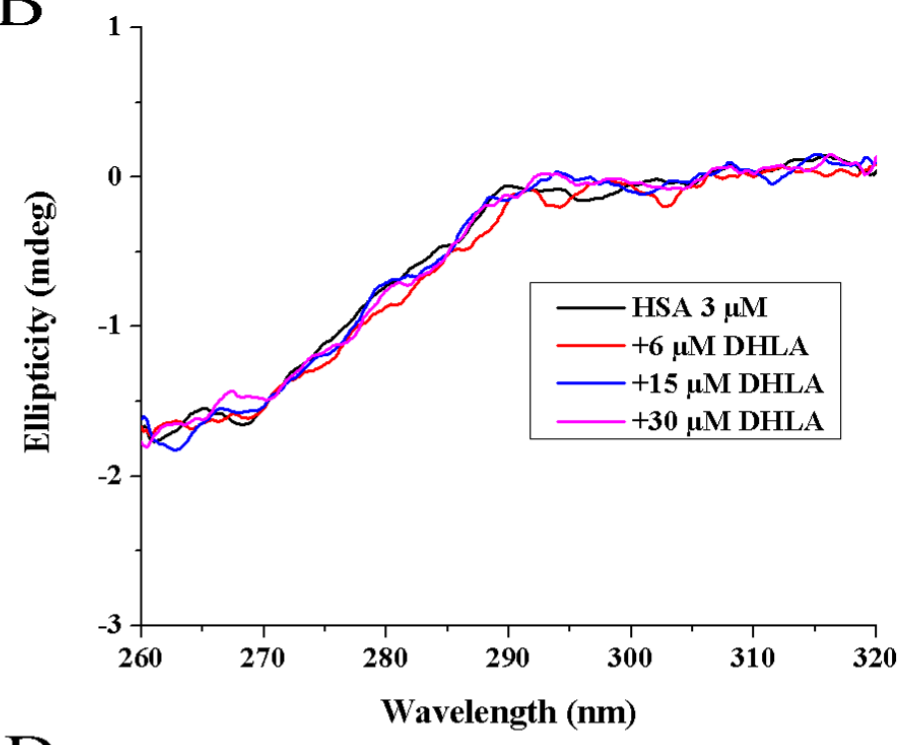

D

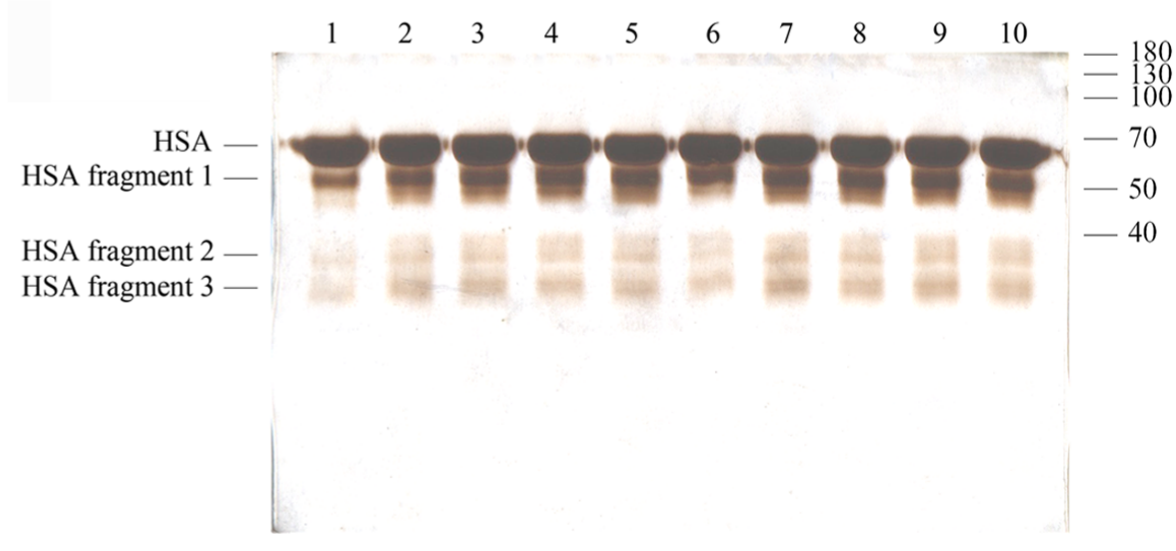


bioRxiv preprint doi: https://doi.org/10.1101/2020.10.16.342121; this version posted October 19,2020 . The copyright holder for this preprint (which was not certified by peer review) is the author/funder. All rights reserved. No reuse allowed without permission.
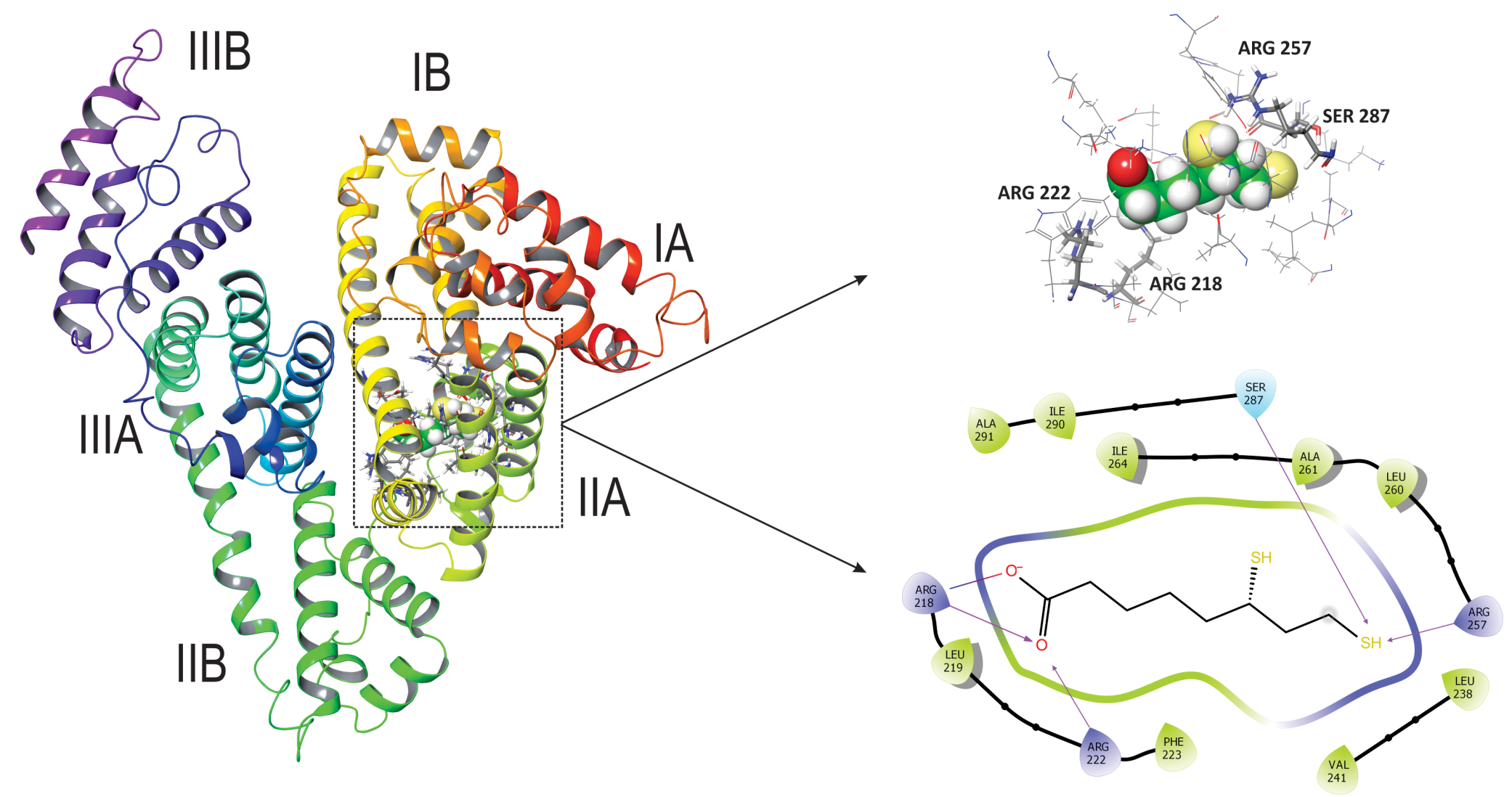

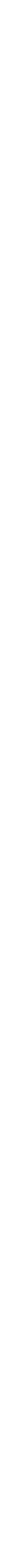